2018

\title{
The Spatial Distribution of Urban Consumer Service Firms: Evidence from Yelp Reviews
}

Brad Humphreys

West Virginia University, Brad.Humphreys@mail.wvu.edu

Josh Matti

West Virginia University, jomatti@mix.wvu.edu

Follow this and additional works at: https://researchrepository.wvu.edu/econ_working-papers

Part of the Regional Economics Commons

\section{Digital Commons Citation}

Humphreys, Brad and Matti, Josh, "The Spatial Distribution of Urban Consumer Service Firms: Evidence from Yelp Reviews" (2018). Economics Faculty Working Papers Series. 9.

https://researchrepository.wvu.edu/econ_working-papers/9 


\section{WestVirginiaUniversity.}

Department of Economics

Working Paper Series

\section{The Spatial Distribution of Urban \\ Consumer Service Firms: Evidence \\ from Yelp Reviews}

Brad R. Humphreys

Josh Matti

Working Paper No. 18-03

This paper can be found at the College of Business and Economics Working Paper Series homepage: 


\title{
The Spatial Distribution of Urban Consumer Service Firms: Evidence from Yelp Reviews
}

\author{
Brad R. Humphreys * \\ Josh Matti ${ }^{\dagger}$ \\ West Virginia University \\ West Virginia University
}

January 29, 2018

\begin{abstract}
A growing literature employs distance-based measures of localization to assess the spatial distribution of firms with a focus on manufacturing across a country. We analyze the spatial concentration of a variety of consumer services firms in the Phoenix, AZ area using geo-referenced Yelp data from over 29,000 establishments. Results from a K-density approach indicate substantial localization and service differentiation among localized firms. Firm concentration varies across service cost and quality; higher quality/cost establishments tend to cluster. Our results further understanding of the modern urban landscape as cities are increasingly centers of consumption.
\end{abstract}

JEL Codes: L80, R12, R32

Keywords: localization, consumer services, consumer city, k-density function, spatial concentration

\footnotetext{
${ }^{*}$ We thank Stephen Billings, Maria Juul Hansen and other participants at the 2017 NARSC Conference in Vancouver, BC, Amelia Biehl and other participants at the 2017 SEA Conference in Tampa, FL, and seminar participants at West Virginia University for valuable comments and suggestions. College of Business \& Economics, Department of Economics, 1601 University Ave., PO Box 6025, Morgantown, WV 26506-6025, USA; Email: brhumphreys@mail.wvu.edu

${ }^{\dagger}$ College of Business \& Economics, Department of Economics, 1601 University Ave., PO Box 6025, Morgantown, WV 26506-6025, USA; Email: jomatti@mix.wvu.edu
} 


\section{Introduction}

Over the last 30 years cities evolved from centers of production to centers of consumption (Glaeser et al., 2001; Glaeser and Gottlieb, 2006). Modern cities attract residents by providing a rich variety of consumer amenities like restaurants, bars, concerts and cultural activities, and other services in relatively small, dense areas. The importance of consumer amenities in cities raises a number of important questions about the distribution of firms across urban areas. Are consumer service firms localized or dispersed within a city? Important consumer search costs exist, and dispersion of service firms in a city may provide lower amenity value than localization.

Little is known about the extent of localization or dispersion of urban consumer service firms. Existing models of consumer service agglomeration predict that firm heterogeneity affects the tendency of consumer service firms to localize (Fischer and Harrington, 1996; Netz and Taylor, 2002; Konishi, 2005; Takahashi, 2013). Understanding spatial location patterns in consumer service firms is important given the rise of consumer cities. Recently developed spatial econometric tools for analyzing the concentration of firms have primarily been applied to firms in manufacturing and business-services industries, not to urban consumer service firms.

Three recent papers analyze locational decisions of consumer service firms using point pattern analyses in the vein of Duranton and Overman (2005). ${ }^{1}$ Mixed evidence on clustering exists in this literature. Nakajima et al. (2012) find that service industry firms, including consumer services such as restaurants and accommodation, medical and health care services, and education, are more clustered than firms in manufacturing sectors at close distances. Zhou and Clapp (2015) find greater dispersion of anchor department stores among new entrants than among existing anchor stores. Billings and Johnson (2016) analyze co-localization of firms in a specific urban area and find evidence of clustering among legal services and data processing firms and dispersion among firms in the accommodations sector. They find that knowledge spillovers, access to transportation, and proximity to consumers explain co-localization across a variety of industries including the consumer services industries in retail trade, arts entertainment and recreation, and accommodation and food services.

This paper contributes to this growing literature by using the K-density function to assess spatial distribution of consumer service firms across a range of consumer service categories in a single urban area. While prior research considers localization by industry type, we also consider localization by cost and quality. Since firms cluster or disperse for multiple reasons and concentration varies across industries, a more complete analysis of the spatial distribution of consumer service providers reveals patterns shaping the landscape of the modern world's increasingly consumer-driven cities.

We use Yelp review data for over 29,000 businesses in the greater Phoenix area to calculate K-densities of localization and dispersion at distances up to 30 kilometers. The results reveal that

\footnotetext{
${ }^{1}$ The Duranton and Overman (2005) kernel density function improves on traditional measures of spatial concentration like the Gini Index or Ellison and Glaeser (1997) indices that rely on pre-defined areas such as counties. By using only Euclidean distances between firms, it overcomes the Modifiable Areal Unit Problem (MAUP) of measures of concentration being sensitive to the selection of the areal units (Marcon and Puech, 2009).
} 
concentration varies across establishment categories, establishment cost and quality, and distances. Establishments in 4 of 15 subcategories in the Yelp food category, 11 of 32 subcategories in the restaurants category, and 19 of 43 subcategories in the shopping category cluster. Despite differences across categories and distances, a general pattern of localization at smaller distances emerges with service differentiation explaining much of the tendency to cluster.

The results also reveal clustering among high quality and high cost consumer service establishments, supporting the predictions in the model developed by Fischer and Harrington (1996). Few previous studies analyze the spatial distribution of establishments by quality and cost, despite the fact that quality and cost are important factors in the provision of consumer services. The variation in densities at different distances underscores the importance of using a point pattern analysis to describe densities for the entire distribution of distances rather than traditional approaches that rely on a single level of aggregation.

\section{Context: Theory and Evidence on Clustering}

A substantial theoretical and empirical literature addresses the tendency of consumer service firms to cluster. This literature appears in both urban economics and industrial organization. The theoretical literature is extensive, and different assumptions can generate predictions along the entire spectrum from extreme clustering to extreme dispersion (Netz and Taylor, 2002). The empirical literature tends to focus on specific industries like retail gasoline or arts and culture firms.

\subsection{Theory}

Much of the theoretical literature on firm clustering focuses on manufacturing with an emphasis on external scale economies and location decisions made by firms. Consumer service firms differ from manufacturing firms in several ways. Consumer service providers cater to households, not other businesses, generally cannot store their output, and cannot take advantage of scale economies to the extent that manufacturing firms can. Most models of agglomeration focus on manufacturing, not consumer service providers. However, several models address the tendency of consumer service firms to localize. ${ }^{2}$

In general, consumer service providers face offsetting incentives to locate near to one another or to spatially differentiate (Netz and Taylor, 2002). All consumer service providers want to locate near large concentrations of consumers, for example in urban areas. Locating close to competitors allows spatially clustered competitors to share a common pool of customers that increases with the number of clustering firms, a market size effect that encourages clustering. However, locating close to competitors increases price competition among firms, a market share effect which tends to push firms apart. While price competition tends to spatially differentiate firms, competing firms can still

\footnotetext{
${ }^{2}$ Incentives for consumer service firms to localize from the perspective of consumers can be motivated by the trip-chaining model of consumer shopping developed by Anas (2007).
} 
cluster and enjoy market size benefits if they can sufficiently differentiate their services by quality. These quality differentiated services tend to be expensive (Schuetz and Green, 2014). Consumer uncertainty about tastes or product quality also enhances clustering (Konishi, 2005; Takahashi, 2013).

The model developed by Fischer and Harrington (1996) motivates the spatial analysis of consumer goods and service providing firms by emphasizing the importance of consumer search and product characteristics. This model includes costly search by consumers and entry by firms that make pricing decisions and sell similar, but differentiated products in either a localized geographic cluster or a dispersed periphery where firms are spread uniformly over space. The inclusion of consumer search and product differentiation makes the model applicable to many consumer services firms like art galleries, jewelery stores or car dealers.

Firms are distinguished by the specific variety they sell, which is drawn from a set of possible varieties. Consider a general category of consumer service firms like restaurants. These firms sell meals, and a large set of possible meal varieties exists. Each restaurant sells a specific variety of meal that is identified by a specific consumer willingness to pay for that variety. The degree of heterogeneity of services provided by firms in this model is reflected by the difference between the largest consumer willingness to pay for a specific variety and the smallest consumer willingness to pay. In the case of restaurants, this interval represents the difference in cost between a lavish meal in a fine dining establishment and the cost of a meal from a food truck or fast food establishment.

Consumers differ in their preference for varieties of goods services, reflected by their willingness to pay, and in their search costs. Search costs on the periphery are higher than search costs in a cluster, and once in a cluster consumers can search all firms located there costlessly.

This model makes specific predictions about the effect of product heterogeneity, and specific characteristics of the services provided, on the likelihood that firms cluster and the equilibrium prices in clusters and the periphery. In particular, the model predicts that the likelihood that firms cluster increases with heterogeneity in services provided by firms and with the size of the consumer expenditure on the service. In both cases, heterogeneity in services provided and heterogeneity in the cost of the service affect the tendency of firms to locate in close proximity to one another.

Humphreys and Zhou (2015) take a different approach to modelling the tendency of consumer service providing firms to localize, emphasizing the importance of monopolistic competition, product variety, travel to consumption centers, and local price effects along the lines of the model developed by Dixit and Stiglitz (1977) in localization decisions. In this model firms are differentiated by their fixed costs of production and the marginal utility generated from consuming the service produced. The model predicts that firms will enter and produce a service at a consumption center where other firms localize depending on the ratio of the firm's fixed cost of production to the marginal utility generated by the service. The model also contains an equilibrium outcome where firms do not localize at a consumption center and instead locate in dispersed spots.

The model developed by Humphreys and Zhou (2015) predicts that some firms will localize in specific consumption centers in a city, and co-locate with other consumer service producing firms 
if their fixed costs of production are low enough, and/or the marginal utility of consumption of the service produced is high enough. Other firms, with larger fixed costs and/or lower marginal utility will not localize. The mechanism for local agglomeration in this model is the effect of firm entry on the local price level coupled with consumer demand for service variety.

A number of models provide specific predictions about localization of consumer service producing firms. These firms may choose to locate near each other under some conditions, forming localized clusters, or alternatively they may choose to locate far from competing firms, generating a pattern of dispersion. Localization generates agglomeration benefits from increased concentration of consumers in local clusters of service providing firms, but also leads to increased price competition. The ability of firms to differentiate their services along non-price margins like quality tends to offset price competition, leading to clustering. This product differentiation appears to be a key factor in determining clustering of consumer service firms (Picone et al., 2009).

\subsection{Empirical Evidence}

Empirical analysis of the distribution of consumer service firms is not a new research area. An early empirical paper, Smith (1985), uses nearest neighbor analysis to describe the distribution of urban restaurants and finds fast food and regular restaurants more clustered than pizza parlors and doughnut shops. Fischer and Harrington (1996) analyze the geographic distribution of a range of consumer services such as auto dealers, clinics, and supermarkets. They find shoe stores the most localized and movie theaters the least localized. Recent empirical research has not only described patterns of clustering but also sought to identify specific factors driving the spatial distribution of firms.

Netz and Taylor (2002) find that gasoline stations in Los Angeles are dispersed and become more dispersed with increased competition. A one standard deviation increase in the number of gas stations within one mile increases spatial dispersion by $23 \%$. Picone et al. (2009) find that establishments selling alcohol on premises ("onsite"), like bars and restaurants, are more localized than establishments selling alcohol "offsite" like liquor stores, in five US cities. They attribute this pattern to greater service differentiation by onsite establishments. Schuetz and Green (2014) find that, at the census-tract level, Manhattan art galleries are localized with the pattern of localization stable over time. In these studies, the extent of localization can be explained by agglomeration economies, population density, and household income. While all these studies analyze the distribution of consumer services establishments, none use the point pattern analysis popularized by Duranton and Overman (2005).

Although not the first to use point pattern analysis at different geographic scales ${ }^{3}$, Duranton and Overman (2005) has been the most influential. In their analysis of UK manufacturing firms, they find that $52 \%$ of industries are localized with localization occurring at closer distances. They also argue that any good test for spatial concentration must satisfy five criteria: (i) comparability

\footnotetext{
${ }^{3}$ For example, Barff (1987) with manufacturing firms in Cincinnati, Ohio and Marcon and Puech (2003) with manufacturing firms in France.
} 
across industries; (ii) controls for the overall agglomeration of manufacturing; (iii) controls for industrial concentration; and (iv) unbiasedness with respect to scale and aggregation. In addition, a good test should also (v) give an indication of the significance of the results. (p. 1079)

Subsequent research on spatial concentration using tests satisfying all five criteria tend to follow Duranton and Overman (2005) by analyzing nationwide manufacturing concentration. Duranton and Overman (2008) find that co-localization of vertically-linked manufacturing industries in the UK occurs at larger distances (around 150km) but not at smaller distances. Behrens and Bougna (2015) find that roughly half of Canadian manufacturing firms are localized, although the extent of localization has declined over the last decade. In addition to describing the distribution of manufacturing industries, there is at least one study using a continuous point pattern approach to test for what causes localization.

Ellison et al. (2010) determine that the co-localization of manufacturing industries in the United States can be explained by labor market pooling, knowledge spillovers, and a tendency to locate near customers or suppliers. While manufacturing has been the focus, Barlet et al. (2013) also analyze business-oriented services in France, finding that services cluster at short distances (less than $4 \mathrm{~km}$ ). While all these studies use point pattern analyses, they focus on the countrywide distribution of manufacturing or business services while this paper focuses on consumer services within an urban area.

Billings and Johnson (2016) develop a novel measure of co-location, analyze co-location decisions, and assess the importance of different sources of agglomeration across a wide variety of establishments in a single urban area, Denver Colorado. The focus on a single urban area motivates this analysis. They find relatively small rates of co-localization, between $3.3 \%$ and $5.4 \%$, between pairs of four-digit NAICS code industries within two-digit NAICS code industries in consumer services (Art, entertainment, and recreation and Accommodation and food services). They also identify natural advantage, and to a lesser extent consumption externalities, as important factors explaining observed co-location.

Given our focus on the greater Phoenix metropolitan area, studies assessing the distribution of firms in this area are also relevant. Four studies using point pattern analyses of Phoenix establishments exist; only two focus on consumer services and none account for the overall concentration of industry in the area. Ó hUallacháin and Leslie (2007) find that all 11 of the producer services studied are clustered at all levels between 0-20 miles. Ó hUallacháin and Leslie (2009) find that all 9 of the manufacturing industries are clustered with transportation networks shaping the patterns of localization.

Leslie and Ó hUallacháin (2006) use nearest neighbor analysis and location quotients to describe the clustering of 10 industries including the consumer services industries of retail, health care and social assistance, and entertainment and accommodation. While all industries are localized in all areas of Phoenix, the localization of each industry varies across the CBD and various suburban sub-centers. Most recently, Ó hUallacháin and Leslie (2013) find varying levels of localization and co-localization across retail. However, their analysis focuses on retail instead of all consumer 
services, uses data from 2004, and does not account for overall retail concentration.

The previous empirical literature on localization focuses on specific industries and finds evidence of both localization and dispersion, depending on characteristics of the firms, products, and services. In consumer service industries, localization can generally be attributed to the ability of firms to differentiate the services provided.

\section{Data}

Although continuous point pattern measures of concentration have been used in economics for over a decade, empirical studies remain rare. One reason for this is data availability. The Kdensity approach requires both data on large numbers of establishments that can be grouped and precisely defined establishment locations. Many public data sources do not meet these requirements. The data made available through the Yelp Dataset Challenge fulfills these requirements. These data sets contain information on thousands of establishments and a wide variety of establishment characteristics including summary information about cost and service quality, and the exact latitude and longitude for nearly 86,000 rated establishments across 10 cities in 4 countries.

Using Yelp data to analyze localization of urban businesses requires that these data accurately reflect the actual number and location of establishments. Glaeser et al. (2017) assess the reliability of Yelp data by comparing establishments covered in Yelp to County Business Patterns (CBP) data across the United States. Glaeser et al. (2017) conclude that establishment coverage in Yelp data is similar to CBP data in retail, leisure and hospitality industries in dense urban areas at the ZIP code level. In some sectors, for example restaurants, Yelp data may contain firms with small numbers of employees that are not included in CBP data.

To assess the Yelp coverage of food, restaurant, and shopping establishments in Phoenix, we compare the Yelp data to 2015 CBP data at the zip code level. CBP classifies establishments by the North American Industry Classification System (NAICS). Following Glaeser et al. (2017), we define NAICS restaurant codes as Full-Service Restaurants (NAICS 722511), Limited-Service Restaurants (722513), Cafeterias, Grill Buffets, and Buffets (722514), and Snack and Nonalcoholic Beverage Bars (722515).

In Phoenix zip codes containing at least one Yelp 'Restaurant' establishment, the CBP data contain 6,259 restaurants based on establishment counts from those four NAICS codes. The Yelp data contain 6,263 restaurants, slightly more than the CBP count. The comparable numbers for CBP 'Food' and 'Shopping' establishments in Phoenix are 2,536 and 5,125. ${ }^{4}$ The Yelp data contain 2,661 food establishments and 4,437 shopping establishments. The Yelp data in all three categories accurately reflect CBP establishment counts.

\footnotetext{
${ }^{4}$ NAICS codes mapped in to the Yelp 'Food' category include Mobile Food Services (722330), Snack and Nonalcoholic Beverage Bars (722515), Caterers (722320), Gasoline Stations with Convenience Stores (447110), and NAICS sub-sector 445 (Food and Beverage Stores). NAICS codes mapped into the Yelp 'Shopping' category include Furniture and Home Furnishings Stores (442), Electronics and Appliance Stores (443), Building Material and Garden Equipment and Supplies Dealers (444), Clothing and Clothing Accessories Stores (448), Sporting Goods, Hobby, Musical Instrument, and Book Stores (451), and Miscellaneous Store Retailers (453).
} 
The Phoenix metro area, which includes several suburbs such as Chandler, Mesa, and Scottsdale, has Yelp data containing information on 31,074 total establishments, the largest number of establishments in any of the Yelp Challenge data sets. In order to restrict the analysis to Phoenix and its closest suburbs, we drop observations for the 1909 establishments located farthest away from the city center. The remaining 29,165 establishments are all located within the rectangular area shown in Figure 1. This area is $74 \mathrm{~km}$ from east to west and $62 \mathrm{~km}$ from north to south, and contains the majority of the greater Phoenix metropolitan area. The density of establishments in the sample generally follows the local population density, with the number of establishments decreasing with distance from the city center.

\section{[Figure 1 about here.]}

Each Yelp establishment is associated with specific descriptors for the services provided. These descriptors take the form of category and sub-category types describing the goods and services provided by each establishment as well as other establishment characteristics. These descriptors are designed to improve users ability to search for, and become informed about, specific establishments. Establishments are grouped into nearly 1000 categories, sub-categories, and sub-sub-categories. Establishments typically possess one primary category and multiple subcategories. For example, the popular Subway sandwich shop is classified in the category Restaurant and further identified by two sub-categories: Fast Food and Sandwiches. However, some establishments possess multiple primary categories. For example, restaurants featuring live music at night may be classified in the Restaurants and Nightlife primary categories.

The Yelp data also contain information on two additional important characteristics of consumer services not found in other data sources: average transaction cost, reflected in a number of dollar signs associated with each establishment and service quality, reflected in a star rating system. Yelp transaction cost information is determined by average cost as reflected in the number of dollar signs associated with each establishment. In the Yelp cost descriptors, one dollar sign $(\$)$ refers to an average transaction cost per person under $\$ 10$. Two dollar signs (\$\$) refers to an average transaction cost between $\$ 11$ and $\$ 30$. Three dollar signs $(\$ \$ \$)$ refers to an average transaction cost between $\$ 31$ and $\$ 60$ and four dollar signs $(\$ \$ \$ \$)$ refers to an average transaction cost over $\$ 61$.

Yelp information on establishment quality come from customer reviews. Customers provide a written review of their experience and a rating of between 1 star (worst) and 5 stars (best) of their experience at the establishment. Yelp publishes the average number of stars each establishment receives in their customer reviews in half-star increments. The number of underlying reviews can vary substantially from a handful to hundreds of customer reviews.

Table 1 shows primary categories, the number of establishments in each primary category in the Phoenix area, and a representative sample of the sub-categories from the 16 primary categories defining the consumer services industry in the Phoenix Yelp data. The total sample from the Phoenix area contains 34,566 establishments and 16 primary categories. Again, some establishments 
fall under multiple primary categories. Our analysis focuses on establishments in three primary categories: food, restaurants, and shopping. We focus on food, restaurants, and shopping because these categories reflect the most common consumer activities. We further restrict the analysis to food, restaurants, and shopping subcategories with 50 or more establishments, resulting in 15 food subcategories, 32 restaurant subcategories, and 43 shopping subcategories.

[Table 1 about here.]

Additionally, price information will be used to analyze establishment localization by service cost. We do not know of any other paper analyzing localization of consumer service firms by price. Not all Yelp-rated establishments have price information in the data set. For example, only $13.63 \%$ of establishments in the health \& medical, $7.46 \%$ in the automotive, and $7.40 \%$ in the home services primary category contain price information. Price information in the primary categories analyzed here is widespread. $90.71 \%$ of establishments in shopping, $96.55 \%$ in restaurants, and $97.07 \%$ in food establishment primary categories have price information.

Figure 2 shows the distribution of the Yelp cost descriptors for consumer services as a whole, as well as the food, restaurants, and shopping primary categories. Within consumer services, most establishments have either one or two dollar signs, indicating that the average transaction at these establishments would be under $\$ 30$. A majority of establishments in the food and restaurant primary categories have one dollar sign; a majority of establishments in the shopping primary category have two dollar signs.

[Figure 2 about here.]

Figure 3 shows the distribution of establishment quality measures, in terms of the average number of stars each establishment gets in customer reviews. The distribution of star ratings skews right overall; the modal consumer services firm gets 5 stars, on average and very few consumer service establishments get below 2.5 stars, on average. This probably reflects exits by low quality businesses. Establishment quality measures in the food, restaurants, and shopping primary categories also skew right, although there are relatively few five star establishments in the food and restaurants primary categories.

[Figure 3 about here.]

\section{Methodology}

We follow Duranton and Overman's (2005) three-step process for describing the spatial distribution of establishments. First, we estimate a kernel density function of the distribution of all pairwise distances of establishments within the same consumer services category. Second, in order to assess the statistical significance of localization or dispersion in these firms, we construct counterfactuals where establishments in the particular industry under consideration are randomly distributed 
across sites occupied by firms in the consumer services industry as a whole. Third, based on the counterfactuals, confidence intervals are constructed and measures of localization and dispersion calculated based on these confidence intervals.

To estimate kernel densities, for each category $c$ with $n$ establishments, we calculate the Euclidean distance between each pair of establishments within category $c$. This results in $\frac{n(n-1)}{2}$ pairwise distances for category $c$. These distances are then kernel-smoothed to estimate the distribution of pairwise distances. The estimator of the density of pairwise distances at distance $d$ (henceforth K-density) is:

$$
\hat{K}_{c}(d)=\frac{1}{n(n-1) h} \sum_{i=1}^{n-1} \sum_{j=i+1}^{n} f\left(\frac{d-d_{i j}}{h}\right)
$$

where $d_{i j}$ is the Euclidean distance between establishment $i$ and $j, h$ is the bandwidth, and $f$ is the kernel function. The bandwidth $h$ is the width of the bins used to describe the true distribution of bilateral distances. Using a correct bandwidth is important to avoid over-smoothing or undersmoothing. We follow Duranton and Overman (2005) by using the optimal bandwidth of Silverman (1986) as this is the default in the R package dbmss (Marcon et al., 2015). We calculate K-densities at $100 \mathrm{~m}$ intervals up to $30 \mathrm{~km}$. The maximum of $30 \mathrm{~km}$ is selected because Duranton and Overman (2005) use the median pairwise distance as their maximum and the median pairwise distance with our observations is around $32 \mathrm{~km}$.

Constructing counterfactuals is the second step. All consumer services establishments are randomly relabeled still using $n$ establishments for category $c$. K-densities are then calculated as in the first step and 1000 simulations are run to create the set of counterfactuals. This process controls for the distribution of the consumer services industry as a whole. Therefore, localization of category $c$ means localization relative to other consumer services and not absolute spatial concentration.

The third step is to evaluate statistical significance with global confidence bands. $\bar{K}_{c}(d)$ is the upper global confidence band while $\underline{\mathrm{K}}_{c}(d)$ is the lower global confidence band. $95 \%$ of the simulations between $0-30 \mathrm{~km}$ lie below the upper band while $95 \%$ lie above the lower band. A category is considered localized if $\hat{K}_{c}(d)>\bar{K}_{c}(d)$ for at least one $d \in[0,30000]$. The classification for dispersion is less straightforward because by construction K-densities sum to one so that categories highly localized at close distances will by default most likely be dispersed at larger distances. We follow Duranton and Overman (2005) by defining a category as dispersed if $\hat{K}_{c}(d)>\underline{K}_{c}(d)$ for at least one $d \in[0,30000]$ and the category is not localized. Therefore, the index of localization for category $c$ at distance $d$ is:

$$
\Gamma_{c}(d)=\max \left(\hat{K}_{c}(d)-\bar{K}_{c}(d), 0\right)
$$

while the index of dispersion is: 


$$
\Psi c(d)= \begin{cases}\max \left(\underline{\mathrm{K}}_{c}(d)-\hat{K}_{c}(d), 0\right) & \text { if } \sum_{d=0}^{30,000} \Gamma_{c}(d)=0 \\ 0 & \text { otherwise }\end{cases}
$$

The indexes for localization and dispersion across the entire range are

$$
\Gamma_{c}=\sum_{d=0}^{30,000} \Gamma_{c}(d) \text { and } \Psi_{c}=\sum_{d=0}^{30,000} \Psi_{c}(d) .
$$

Graphically, establishments in a subcategory are localized when the K-density lies above its upper global confidence band. Establishments in a subcategory are dispersed when the K-density lies below its lower global confidence band and never lies above its upper global confidence band. We estimate K-densities and upper/lower confidence bands for all subcategories in the food, restaurants, and shopping primary categories in the Yelp data for the Phoenix area.

A comparison of K-densities and the upper/lower confidence bands can identify subcategories where establishments are localized, dispersed, or spatially distributed in the same way as all consumer service establishments in the area. Figure 4 illustrates the relationship between the K-density estimates and confidence bands for establishments in four different subcategories: bakeries, Indian restaurants, department stores, and antiques. From Figure 4, firms in the bakeries subcategory are neither localized nor dispersed, as the K-density for this subcategory lies entirely in the Phoenix confidence band at all distances. Firms in the Indian restaurants subcategory are localized at close distances, less than ten kilometers, as the K-density lies above the upper confidence band from 0-9500m. Establishments in the department stores subcategory are dispersed, as the K-density lies below the confidence bands and never lies above the confidence bands. Firms in the antiques classification are localized even though the K-density falls below the lower global confidence band at larger distances.

[Figure 4 about here.]

To provide intuition into the patterns of location associated with localized and dispersed subcategories, the left panel of Figure 5 displays the location of all 53 Indian restaurants in the Phoenix area. Consistent with the K-density plot in Figure 5, a number of clusters of Indian restaurants at close distances can clearly be seen in Figure 5.

The right panel of Figure 5 shows the location of all 266 department stores in the Phoenix area. Consistent with the K-density plot in Figure 4, some clusters of department stores at close distances can be seen, but many isolated establishments also exist, reflecting an absence of localization or dispersion at close distances. However, clusters tend not to locate near one another, and the overall distribution of pairwise distances between department stores in Phoenix is large, which is reflected by dispersion at larger distances on the K-density plot for this subcategory. While visual inspection of maps is illustrative, a formal K-density test is required to determine how different types of consumer services locate relative to the consumer services industry as a whole.

[Figure 5 about here.] 


\section{Results}

We first analyze the spatial distribution of establishments in specific Yelp categories and subcategories in the Phoenix area. These groups of establishments mirror the industries used in previous research on firm location decisions. We then turn to an analysis of the spatial distribution of consumer service establishments by service quality and cost. Previous theoretical research established the role of cost in location decisions (Fischer and Harrington, 1996) but previous empirical analysis generally lacks information on service cost and quality.

\subsection{Results by Establishment Category}

K-density functions and upper/lower confidence bands were estimated for 15 food subcategories, 32 restaurant subcategories, and 43 shopping subcategories using Equation (1). Counterfactuals reflect the location of all consumer service establishments in the Yelp data. ${ }^{5}$ Indexes of localization, from Equation (2), and dispersion, from Equation (3), were also estimated for all subcategories. The results indicate that localization patterns vary across subcategories and distances.

Note that localization could result from limited locations for service providing firms to open. These limitations could come from local zoning laws, local building restrictions, or local geographic features like hills or water. Many measures of localization cannot account for these factors. The Kdensity approach developed by Duranton and Overman (2005) uses the actual spatial distribution of all firms in the area as a counterfactual, and measures localization and dispersion relative to the existing spatial distribution of consumer service firms. The actual spatial distribution will reflect any local conditions that limit the locations where firms can locate.

The location patterns of food establishments differs the least from the overall location of consumer services. In the food category, 8/15 subcategories are neither localized nor dispersed, with 4 categories localized and 3 dispersed. Establishments in the restaurants category are more likely to differ spatially from consumer services as a whole; 11 subcategories contain localized establishments, 8 contain dispersed establishments, and 13 contain neither localized nor dispersed establishments. Establishments in the shopping category are the most likely to be localized; 19 subcategories contain localized establishments, 7 contain dispersed establishments, and 17 neither localized nor dispersed establishments.

To understand broad location patterns across distances and subcategories, Figure 6 displays the fraction of categories with significant localization or dispersion at each distance from 0-30,000m. The overall pattern in food, restaurants, and shopping shows the prevalence of localization decreasing with distance, while the share of dispersed categories remains relatively constant. However, patterns of dispersion vary based upon category. Dispersion decreases with distance for food, remains relatively constant with shopping, and is most common around $15,000 \mathrm{~m}$ for restaurants.

[Figure 6 about here.]

\footnotetext{
${ }^{5}$ An alternative approach - constructing counterfactuals using only the location of restaurants, food establishments and shopping establishments - produced very similar results.
} 
The general pattern of localization decreasing with distance is consistent with the proliferation of shopping centers and polycentrism of Phoenix. Over the last 60 years, establishments have increasingly clustered in shopping centers to profit from more potential customers (Carter, 2009). Shopping centers are densely packed with consumer services at short distances, but two shopping centers are unlikely to locate next to each other. Consistent with this view is Zhou and Clapp's (2015) finding of greater dispersion of department anchor stores among new stores than existing stores. Anchor stores are the foundation of shopping centers so a greater dispersion of anchor stores leads to a greater dispersion across shopping centers. Shopping centers are especially relevant for Phoenix. Leslie and Ó hUallacháin (2006) find considerable co-localization between retail and entertainment establishments, which is unsurprising given the shopping centers and strip malls dotting the sprawling landscape.

Table 2 provides specific information about the most localized subcategories of establishments in food, restaurants, and shopping. Yelp subcategories are specific enough to include competing establishments, but broad enough to include establishments with differentiated services. While price competition leads firms to spatially differentiate or disperse, service differentiation can lead similar firms to cluster. Subcategories that exhibit localization should include firms with the ability to differentiate their services.

[Table 2 about here.]

The most highly localized shopping subcategories include industries already identified in the literature as containing establishments highly differentiated in terms of quality. These include art galleries (Schuetz and Green, 2014) and jewelry and women's clothing (Fischer and Harrington, 1996). Antiques and vintage/consignment clothing would appear to contain establishments that operate along similar lines to art galleries.

Highly localized subcategories in the food category also support the predictions from models of clustering in consumer services. Food trucks exhibit the largest degree of localization in the food category and clearly have very low fixed costs. Recall that the model developed by Humphreys and Zhou (2015) predicts that establishments with low fixed costs will tend to localize. The other three food subcategories with a high degree of localization, beer, wine and spirits, coffee and tea, and specialty foods are establishments that can plausibly differentiate their services in terms of quality or other characteristics. All can specialize in products from different areas of the world (craft beers, European wines, coffees from exotic locations) or in the case of specialty food, cater to customers with different dietary preferences (kosher, halal, gluten-free, etc.). Specialty food establishments, by definition, provide differentiated services, so establishments in this subcategory would be expected to cluster.

The highly localized subcategories in food show similar establishment characteristics. Restaurants have a large number of quality-related features available for differentiation, including service, decor, ambience, regional specialization, menu breadth, and other features. This rich environment for quality differentiation within a restaurant subcategory like American (New) or Mexican provides establishments in these subcategories with ample room to compete on margins other than price. 
Table 3 provides specific information about the most dispersed subcategories in food, restaurants, and shopping. These subcategories contain establishments with relatively few margins on which to differentiate. In the shopping category, department stores carry a wide variety of products like clothing, electronics, furniture, toys, books, sundries, and home goods. Their comprehensive nature provides little flexibility in terms of offerings or quality, and also makes price competition more intense, which leads them to spatially differentiate through dispersion. Drugstores also have little room to diversify, as consumers expect these establishments to carry a standard array of over the counter medications, greeting cards, and seasonal products. Cosmetic stores also carry a standard selection of makeup, lipsticks, creams, lotions, and other beauty products. Mattresses and mobile telephony stores provide commodity products differentiated primarily by brand. In all these subcategories, similarities in the consumer services offered would amplify price competition, leading them to locate farther from one another.

[Table 3 about here.]

In the food category, ice cream and frozen yogurt shops sell homogenous products with little room for variation in quality. Grocery stores and convenience stores face the same problems as department stores and drug stores, in that they must offer a similar array of products and services as their competitors with little room to differentiate.

In the restaurant category, fast food restaurants and pizza shops are highly dispersed. These establishments have little ability to differentiate on quality. Fast food restaurants offer homogenous, low cost, quick meals. Pizza shops frequently offer delivery, which would also tend to disperse locations. Sushi Bars offer a uniform menu of raw fish prepared in a limited number of ways; many have the same seating format.

\subsection{Results by Cost and Quality}

While localization or dispersion by establishment category or subcategory is a reasonable way of analyzing the spatial distribution of consumer services, other factors are likely to affect their location patterns. For example, localization decisions could also depend on the cost of the service provided. High end shopping establishments may cluster together in expensive shopping centers. To explore other potential factors relating to consumer services locations, we consider how consumer services are located by price, category-price, and rating.

The Yelp data contains information about service characteristics that is not present in many other establishment level data sources: dollar sign data that provides information about the cost of consumer services provided and star ratings that provide information about the quality of the services provided based on customer reviews of the businesses. Recall that Fischer and Harrington (1996) characterize differentiation in terms of the variability of consumer willingness to pay. The dollar sign ratings provided by Yelp can be thought of as a proxy for variability in consumer willingness to pay. One dollar sign restaurants have an average cost per person of under $\$ 10$. Two dollar sign restaurants have an average cost per person of between $\$ 11$ and $\$ 30$ per person. These 
restaurants likely provide options for customers to pay less that $\$ 11$, for example by ordering only a cheap entree, salad or appetizer, but also provide the opportunity for customers to spend more on a meal. In this sense, two dollar sign restaurants provide a wider range of willingness to pay for meals than a one dollar sign restaurant, which matches the differentiation measure used by Fischer and Harrington (1996). Therefore, two dollar sign restaurants could be expected to localize more than one dollar sign restaurants.

Table 4 shows location patterns by dollar sign rating for the shopping, food, and restaurants categories. As expected, one dollar sign shopping and restaurants show no pattern of localization. One dollar sign restaurants are more dispersed that the average consumer service firm, and one dollar sign shopping establishments have the same spatial distribution as the overall spatial distribution of consumer service firms in the area. However, two dollar sign shopping and two dollar sign restaurant establishments are localized. This likely reflects more differentiation in the services provided by these more costly establishments.

\section{[Table 4 about here.]}

From Table 4, one dollar sign food establishments are localized, but two dollar sign food establishments are not. Table 2 shows the most localized subcategories in the food category: food trucks, beer, wine, \& spirits, coffee \& tea, and specialty foods. Why might low cost establishments in the food category cluster? Food trucks typically produce a single type of food or dish; even low cost specialty foods can be highly differentiated. Picone et al. (2009) find that offsite alcohol shops were less localized than onsite providers, but provide no information about the degree of localization relative to the overall spatial distribution of consumer service firms in cities. Picone et al. (2009) report that offsite alcohol shops are more localized than elementary schools, which could indicate general localization.

Table 5 provides more detailed information about the spatial distribution of consumer service providers, showing localization and dispersion indexes for selected subcategories by dollar sign rating. One dollar sign coffee \& tea establishments are localized, but two dollar sign establishments are not. This suggests that low cost coffee \& tea shops can generate enough differentiation to overcome costs associated with price competition and localize. This could reflect variation in factors like ambience or variety of specialty drinks offered.

[Table 5 about here.]

The rest of the spatial patterns by establishment cost generally support a relationship between service differentiation and localization. One dollar sign establishments in fashion and American "traditional" restaurants do not localize but two dollar sign establishments localize. Two dollar sign establishments exhibit more variation in consumer willingness to pay, which reflects diversification in the model developed by Fischer and Harrington (1996).

In subcategories with substantial dispersion, grocery stores and drug stores, the tendency to disperse increases with costs. Again, these establishments generally provide a homogenous set of 
consumer products which would make price competition more intense, leading them to locate farther from each other to reduce price competition. Two dollar sign grocery stores and drug stores may have higher markup over marginal cost for consumer staples than one dollar sign establishments, which would lead them to locate even farther apart.

Finally, the Yelp data contain information on the quality of the service provided, as reflected in customer ratings. Increasing the quality of service can be thought of as a way for establishments to generate differentiation in the minds of consumers. At low quality establishments, all customers are treated (equally) badly. High quality establishments may be able to create an impression that they cater to individual customer preferences in some way, making them distinct from other establishments. Also, clustering of high quality establishments may create a "halo" effect where customer perceptions of a high quality of service spill over when customers visit other nearby establishments.

[Table 6 about here.]

Table 6 displays the location patterns for low, medium, and high quality establishments in the food, restaurant, and shopping categories. Low quality is defined as establishments with an average rating of $1,1.5$, or 2 stars; medium quality as establishments with an average rating of 2.5 , 3, or 3.5 stars; high quality establishments have average ratings of 4, 4.5, or 5 stars. Each establishment included in this location analysis contains at least 3 customer reviews; many have hundreds of reviews. High quality establishments for food, restaurants, and shopping are all localized, but medium and low quality establishments tend to be dispersed. Again, this pattern suggests that the quality of service offered is an important component of service differentiation.

\section{Conclusions}

Previous research primarily used the spatial econometrics tools developed in the last decade to understand the spatial distribution of manufacturing and business-services firms. Research using modern methods of spatial distribution analysis developed by Duranton and Overman (2005) to analyze consumer services are rare. We use Yelp data from over 29,000 consumer service establishments to contribute to this growing literature by describing the location patterns of consumer services establishments in the greater Phoenix area.

Localization is generally more prevalent than dispersion in this setting, and service differentiation explains much of the observed clustering of establishments. However, concentration varies across distances, categories of consumer services, cost and quality. The variation in densities at different distances underscores the importance of using a point pattern analysis to describe densities over the entire distribution of distances rather than traditional approaches that rely on a single level of aggregation.

Most previous research does not analyze the spatial distribution of consumer service firms based on cost or quality of the service provided. Our results show substantial clustering among high cost 
and high quality consumer service establishments. This clustering is consistent with the predictions of the model developed by Fischer and Harrington (1996), who define service differentiation based on variability in consumer willingness to pay. The observed differences in localization by cost and quality highlight the importance of considering more than just industry classification as has previously been the focus in the literature.

The widespread clustering across many consumer service providers also has important implications for the development of cities as centers of consumption (Glaeser and Gottlieb, 2006) rather than centers of production. Consumers value the enhanced variety of consumption opportunities in cities, relative to the consumption opportunities in less dense areas. Our results show that within cities, service providing firms tend to cluster, providing urban residents with a rich variety of consumption opportunities in spatially concentrated areas. The observed urban clustering of high cost, high quality shopping and dining opportunities provides new insight into the advantages that cities provide their high income residents, relative to smaller cities and rural areas.

The results in this paper motivate additional research. There are several questions left unexplored. For example, how has consumer services localization changed over time? Does the distribution of consumer services in other cities follow the same trends as in the Phoenix area? What factors explain the localization or dispersion of particular categories of consumer services? Answering these types of questions will shed more light on the changing urban landscapes associated with the rise of the consumer city. 


\section{References}

Anas, A. (2007). A unified theory of consumption, travel and trip chaining. Journal of Urban Economics, 62(2):162-186.

Barff, R. A. (1987). Industrial clustering and the organization of production: a point pattern analysis of manufacturing in Cincinnati, Ohio. Annals of the Association of American Geographers, 77(1):89-103.

Barlet, M., Briant, A., and Crusson, L. (2013). Location patterns of service industries in France: A distance-based approach. Regional Science and Urban Economics, 43(2):338-351.

Behrens, K. and Bougna, T. (2015). An anatomy of the geographical concentration of Canadian manufacturing industries. Regional Science and Urban Economics, 51:47-69.

Billings, S. B. and Johnson, E. B. (2016). Agglomeration within an urban area. Journal of Urban Economics, 91:13-25.

Carter, C. (2009). Review articles: What we know about shopping centers. Journal of Real Estate Literature, 17(2):163-180.

Dixit, A. K. and Stiglitz, J. E. (1977). Monopolistic competition and optimum product diversity. The American Economic Review, 67(3):297-308.

Duranton, G. and Overman, H. G. (2005). Testing for localization using micro-geographic data. The Review of Economic Studies, 72(4):1077-1106.

Duranton, G. and Overman, H. G. (2008). Exploring the detailed location patterns of UK manufacturing industries using microgeographic data. Journal of Regional Science, 48(1):213-243.

Ellison, G. and Glaeser, E. L. (1997). Geographic concentration in US manufacturing industries: a dartboard approach. Journal of Political Economy, 105(5):889-927.

Ellison, G., Glaeser, E. L., and Kerr, W. R. (2010). What causes industry agglomeration? evidence from coagglomeration patterns. The American Economic Review, 100(3):1195-1213.

Fischer, J. H. and Harrington, J. E. (1996). Product variety and firm agglomeration. The RAND Journal of Economics, pages 281-309.

Glaeser, E. L. and Gottlieb, J. D. (2006). Urban resurgence and the consumer city. Urban Studies, 43(8):1275-1299.

Glaeser, E. L., Kim, H., and Luca, M. (2017). Nowcasting the local economy: Using yelp data to measure economic activity. Working Paper 24010, National Bureau of Economic Research.

Glaeser, E. L., Kolko, J., and Saiz, A. (2001). Consumer city. Journal of Economic Geography, $1(1): 27-50$. 
Humphreys, B. R. and Zhou, L. (2015). Sports facilities, agglomeration, and public subsidies. Regional Science and Urban Economics, 54:60-73.

Konishi, H. (2005). Concentration of competing retail stores. Journal of Urban Economics, $58(3): 488-512$.

Leslie, T. F. and Ó hUallacháin, B. (2006). Polycentric Phoenix. Economic Geography, 82(2):167192.

Marcon, E. and Puech, F. (2003). Evaluating the geographic concentration of industries using distance-based methods. Journal of Economic Geography, 3(4):409-428.

Marcon, E. and Puech, F. (2009). Measures of the geographic concentration of industries: improving distance-based methods. Journal of Economic Geography, 10(5):745-762.

Marcon, E., Traissac, S., Puech, F., Lang, G., et al. (2015). Tools to characterize point patterns: dbmss for R. Journal of Statistical Software, 67(3):1-15.

Nakajima, K., Saito, Y. U., and Uesugi, I. (2012). Measuring economic localization: Evidence from Japanese firm-level data. Journal of the Japanese and International Economies, 26(2):201-220.

Netz, J. S. and Taylor, B. A. (2002). Maximum or minimum differentiation? location patterns of retail outlets. Review of Economics and Statistics, 84(1):162-175.

Ó hUallacháin, B. and Leslie, T. F. (2007). Producer services in the urban core and suburbs of Phoenix, Arizona. Urban Studies, 44(8):1581-1601.

Ó hUallacháin, B. and Leslie, T. F. (2009). Postindustrial manufacturing in a sunbelt metropolis: Where are factories located in Phoenix? Urban Geography, 30(8):898-926.

Ó hUallacháin, B. and Leslie, T. F. (2013). Spatial pattern and order in Sunbelt retailing: shopping in Phoenix in the twenty-first century. The Professional Geographer, 65(3):396-420.

Picone, G. A., Ridley, D. B., and Zandbergen, P. A. (2009). Distance decreases with differentiation: Strategic agglomeration by retailers. International Journal of Industrial Organization, 27(3):463473.

Schuetz, J. and Green, R. K. (2014). Is the art market more bourgeois than bohemian? Journal of Regional Science, 54(2):273-303.

Silverman, B. W. (1986). Density estimation for statistics and data analysis, volume 26. CRC press.

Smith, S. L. (1985). Location patterns of urban restaurants. Annals of Tourism Research, 12(4):581602. 
Takahashi, T. (2013). Agglomeration in a city with choosy consumers under imperfect information. Journal of Urban Economics, 76:28-42.

Zhou, T. and Clapp, J. M. (2015). The location of new anchor stores within metropolitan areas. Regional Science and Urban Economics, 50:87-107. 
Figure 1: Spatial Distribution of Consumer Services Establishments in Phoenix

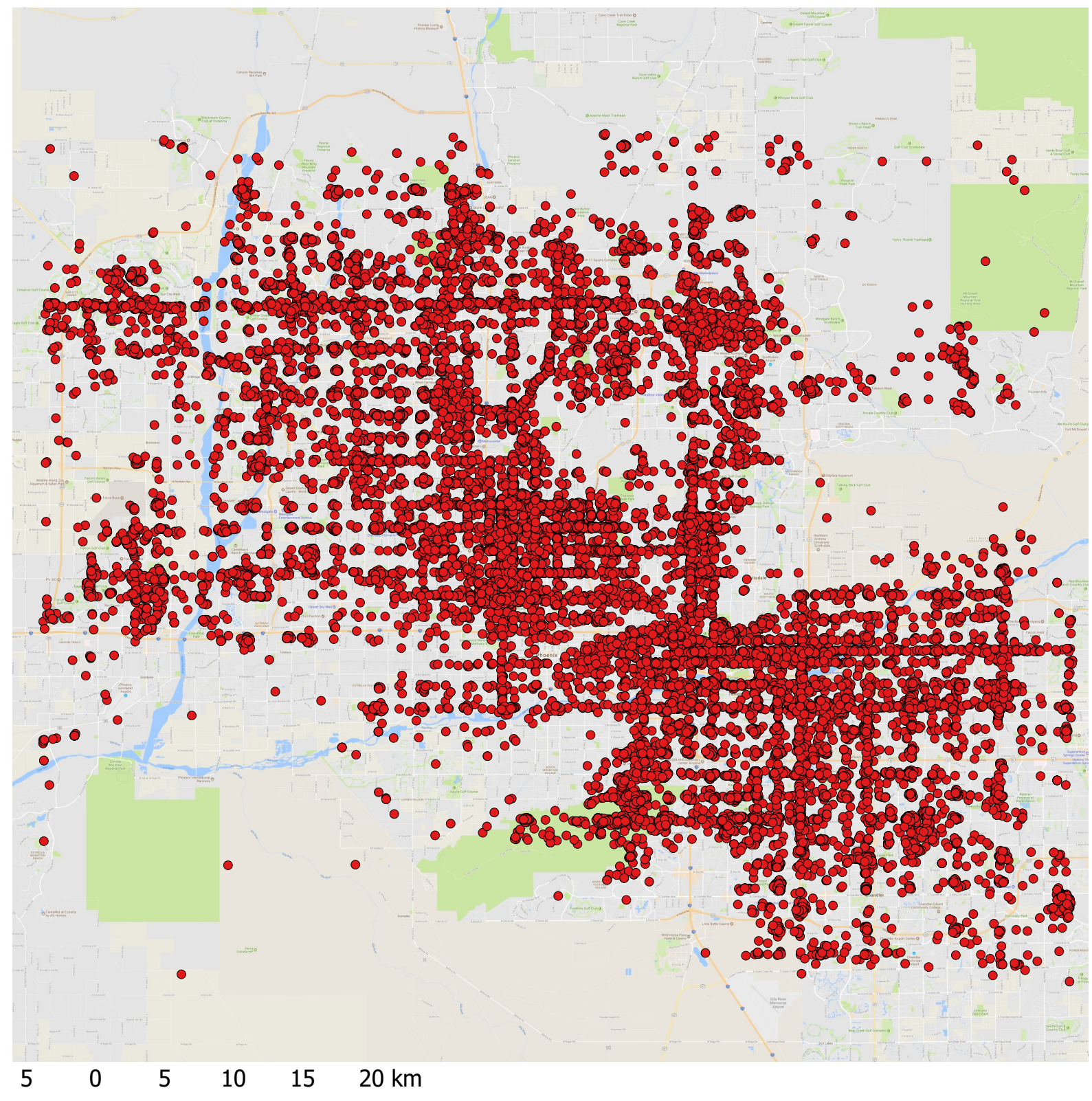


Figure 2: Price (Dollar Sign) Distributions by Primary Category
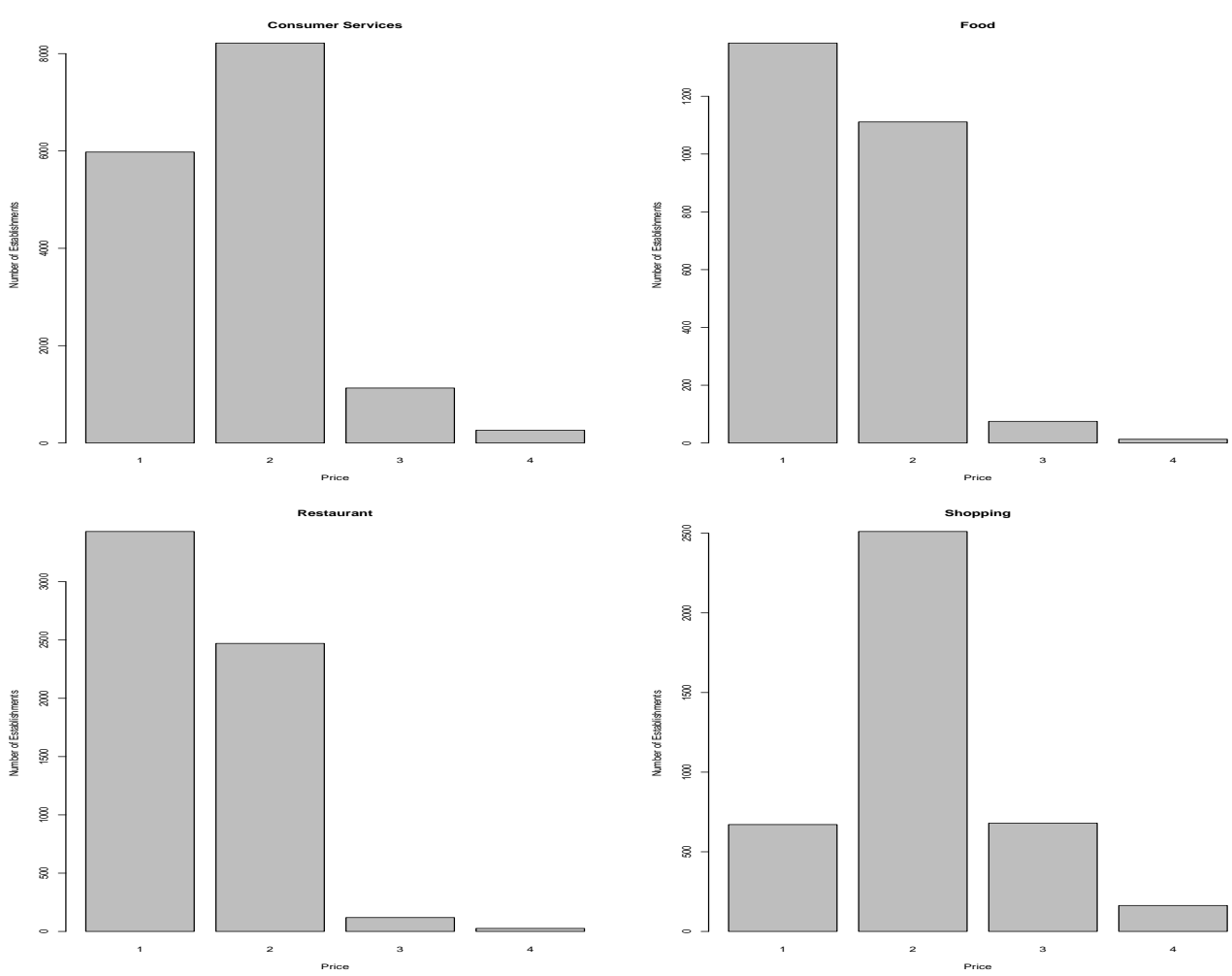
Figure 3: Establishment Quality (Star) Distributions by Primary Category
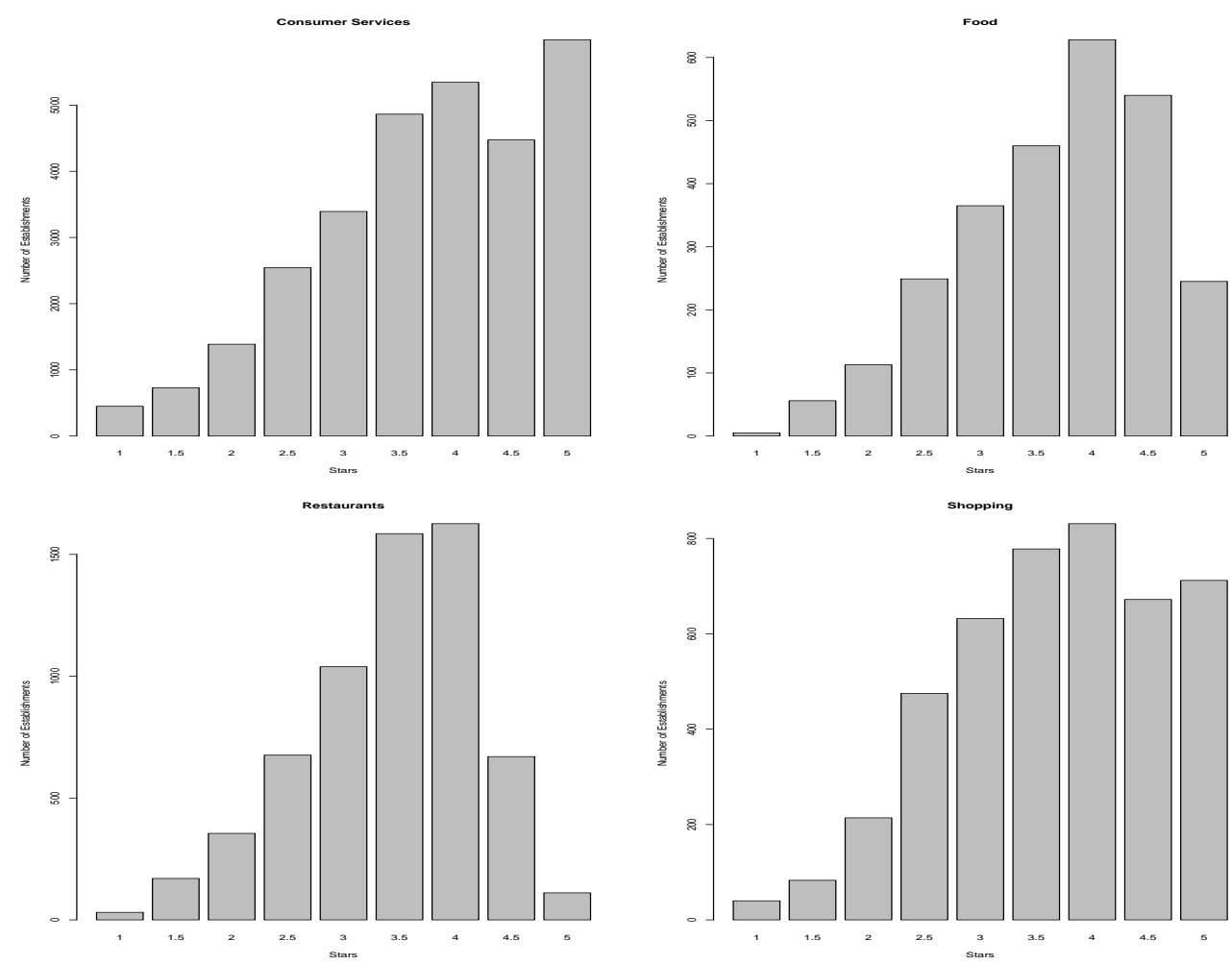
Figure 4: K-densities for Four Illustrative Categories
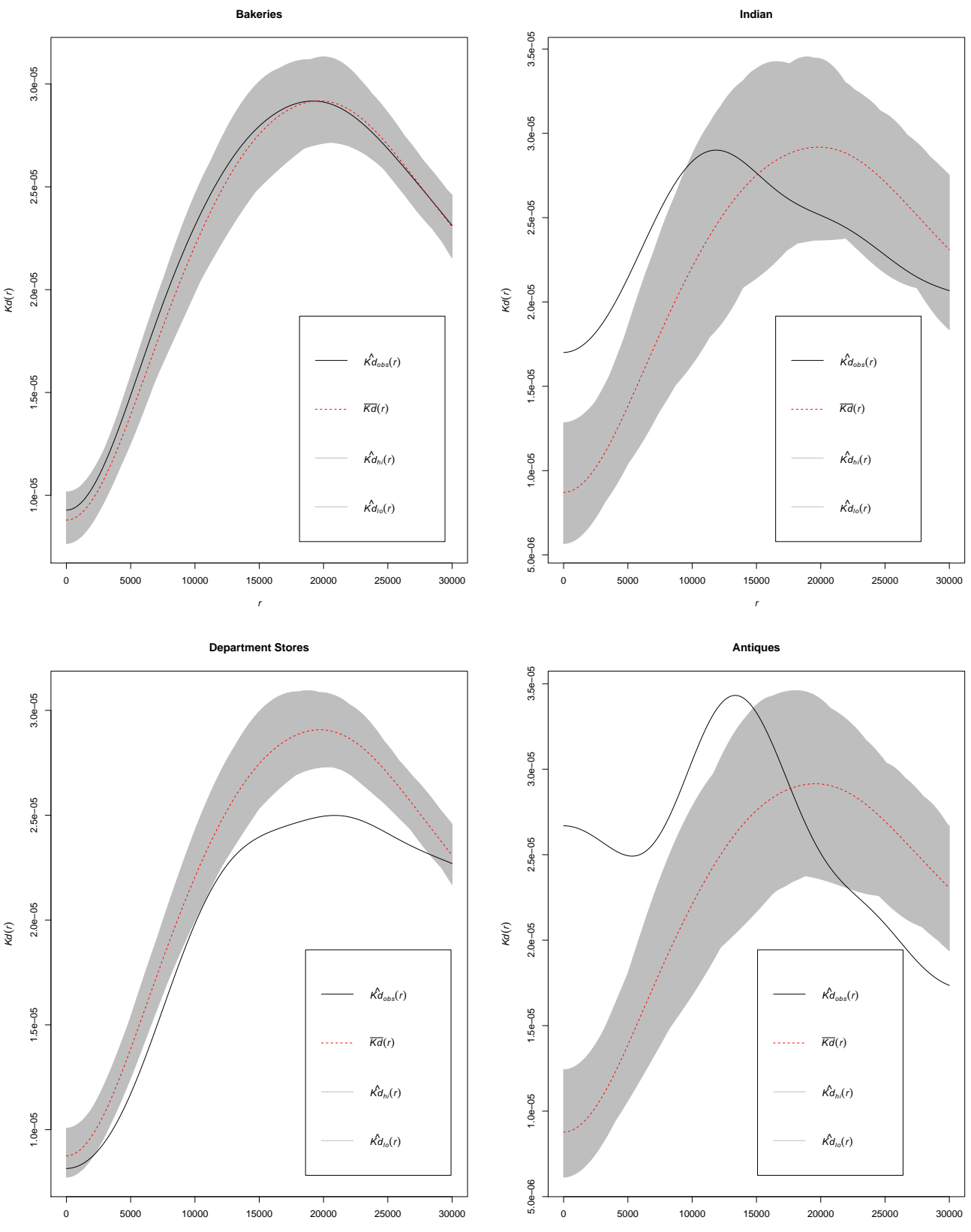
Figure 5: Localization and Dispersion
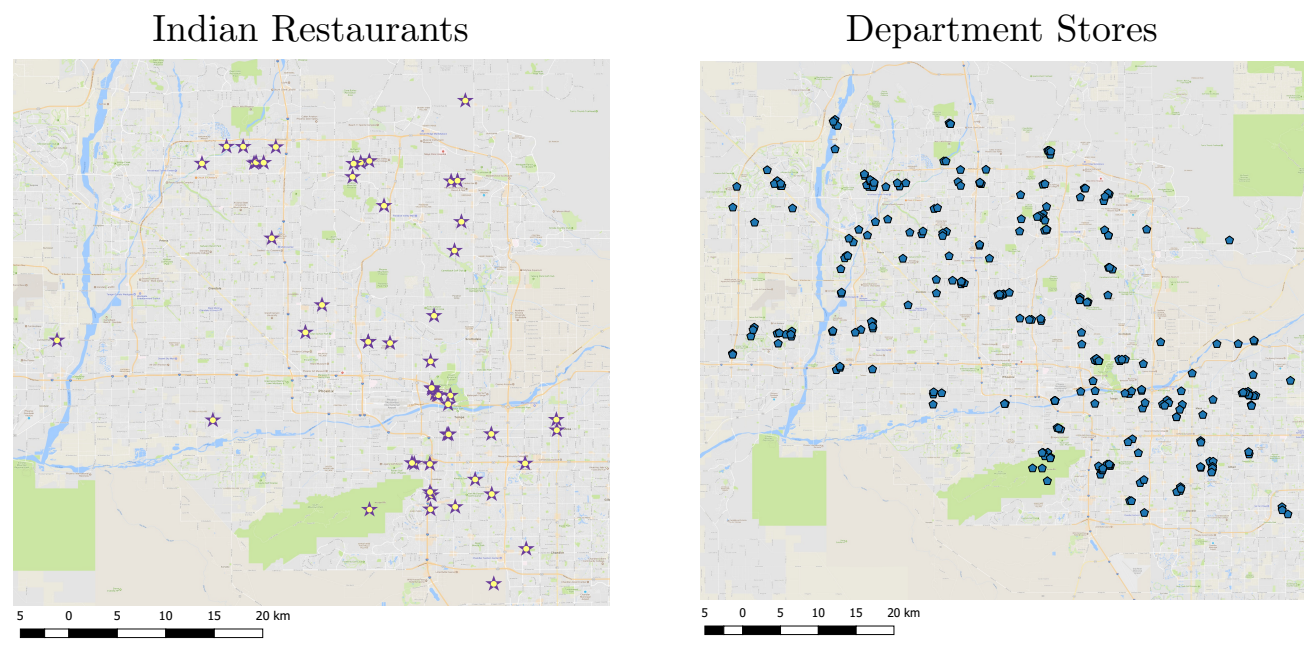
Figure 6: Share of Localized and Dispersed Categories
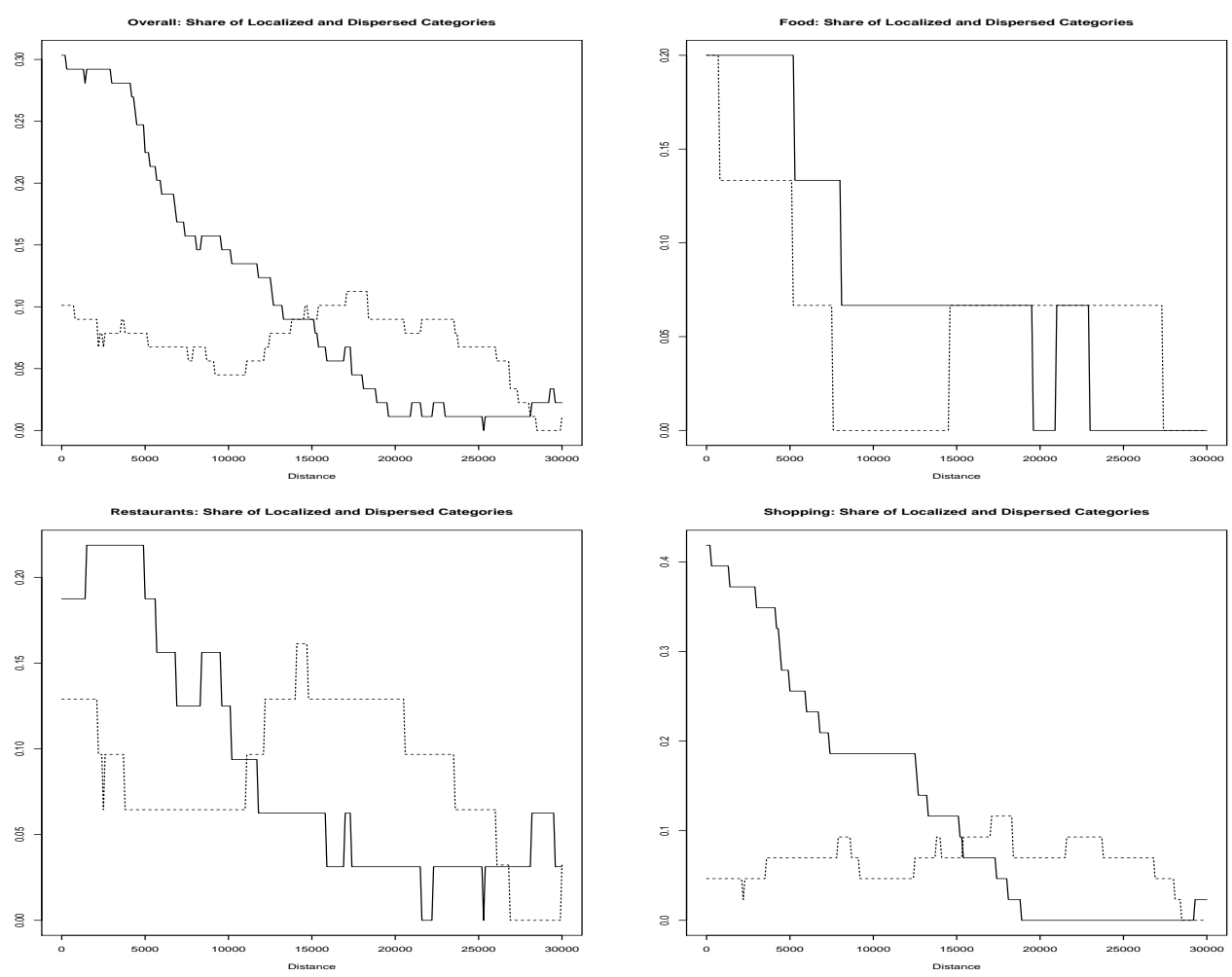
Table 1: Yelp Consumer Service Categories

\begin{tabular}{|c|c|c|}
\hline Primary Category & $\mathrm{N}$ & Sample Subcategories \\
\hline Active Life & 1,234 & $\begin{array}{l}\text { Fitness \& Instruction, Golf, Laser Tag, Parks, Swimming } \\
\text { Pools }\end{array}$ \\
\hline $\begin{array}{l}\text { Arts \& Entertain- } \\
\text { ment }\end{array}$ & 528 & $\begin{array}{l}\text { Art Galleries, Cinema, Museums, Opera \& Ballet, Perform- } \\
\text { ing Arts }\end{array}$ \\
\hline Automotive & 2,332 & $\begin{array}{l}\text { Body Shops, Car Dealers, Car Wash, Gas \& Service Stations, } \\
\text { Parking }\end{array}$ \\
\hline Beauty \& Spas & 3,061 & Barbers, Day Spas, Hair Salons, Piercing, Tanning \\
\hline $\begin{array}{l}\text { Event Planning \& } \\
\text { Services }\end{array}$ & 982 & $\begin{array}{l}\text { Caterers, Party Supplies, Photographers, Wedding Planning, } \\
\text { Valet Services }\end{array}$ \\
\hline Financial Services & 528 & $\begin{array}{l}\text { Banks \& Credit Unions, Financial Advising, Insurance, In- } \\
\text { vesting, Tax Services }\end{array}$ \\
\hline Food & 2,661 & Bakeries, Breweries, Coffee \& Tea, Desserts, Grocery \\
\hline Health \& Medical & 3,104 & $\begin{array}{l}\text { Counseling \& Mental Health, Dentists, Doctors, Medical } \\
\text { Centers, Pharmacy }\end{array}$ \\
\hline Home Services & 3,164 & $\begin{array}{l}\text { Carpeting, Electricians, Home Cleaning, Plumbing, Tree Ser- } \\
\text { vices }\end{array}$ \\
\hline Hotels \& Travel & 753 & Airports, Car Rental, Hotels, Tours, Transportation, \\
\hline Local Services & 1,907 & $\begin{array}{l}\text { Child Care \& Day Care, Community Service/Non-Profit, IT } \\
\text { Services \& Computer Repair, Pest Control, Self Storage }\end{array}$ \\
\hline Nightlife & 993 & Bars, Comedy Clubs, Dance Clubs, Karaoke, Pool Halls \\
\hline Pets & 746 & $\begin{array}{l}\text { Animal Shelters, Pet Adoption, Pet Services, Pet Stores, Vet- } \\
\text { erinarians, }\end{array}$ \\
\hline $\begin{array}{l}\text { Professional } \\
\text { vices }\end{array}$ & 554 & Accountants, Architects, Lawyers, Marketing, Web Design \\
\hline Real Estate & 830 & $\begin{array}{l}\text { Apartments, Mobile Home Dealers, Mortgage Brokers, Prop- } \\
\text { erty Management, Real Estate Agents }\end{array}$ \\
\hline Restaurants & 6,259 & American (Traditional), Fast Food, Chinese, Italian, Seafood \\
\hline Shopping & 4,437 & $\begin{array}{l}\text { Arts \& Crafts, Department Stores, Electronics, Fashion, } \\
\text { Sporting Goods }\end{array}$ \\
\hline
\end{tabular}


Table 2: Most Highly Localized Subcategories

\begin{tabular}{llcc}
\hline Category & Subcategory & Establishments & Localization Index \\
\hline Shopping & Art Galleries & 57 & 0.1271 \\
Shopping & Antiques & 59 & 0.0921 \\
Shopping & Used, Vintage \& Consignment & 96 & 0.0509 \\
Shopping & Jewelry & 225 & 0.0443 \\
Shopping & Women's Clothing & 404 & 0.0212 \\
\hline Food & Food Trucks & 92 & 0.0881 \\
Food & Beer, Wine \& Spirits & 116 & 0.0187 \\
Food & Coffee \& Tea & 551 & 0.0025 \\
Food & Specialty Food & 274 & $<.0001$ \\
\hline Restaurants & American (New) & 431 & 0.0330 \\
Restaurants & Indian & 53 & 0.0262 \\
Restaurants & Vegetarian & 90 & 0.0244 \\
Restaurants & Mexican & 1007 & 0.0080 \\
Restaurants & Cafes & 178 & 0.0063 \\
\hline
\end{tabular}


Table 3: Most Highly Dispersed Subcategories

\begin{tabular}{llcc}
\hline Category & Subcategory & Establishments & Dispersion Index \\
\hline Shopping & Department Stores & 266 & 0.0247 \\
Shopping & Drugstores & 327 & 0.0212 \\
Shopping & Cosmetics \& Beauty Supply & 306 & 0.0035 \\
Shopping & Mattresses & 101 & 0.0016 \\
Shopping & Mobile Phones & 187 & 0.0015 \\
\hline Food & Ice Cream \& Frozen Yogurt & 292 & 0.0085 \\
Food & Grocery & 509 & 0.0014 \\
Food & Convenience Stores & 320 & $<.0001$ \\
\hline Restaurants & Fast Food & 1181 & 0.0245 \\
Restaurants & Pizza & 812 & 0.0109 \\
Restaurants & Sushi Bars & 166 & 0.0059 \\
Restaurants & Chinese & 409 & 0.0047 \\
Restaurants & Tex-Mex & 127 & 0.0013 \\
\hline
\end{tabular}


Table 4: Location Patterns by Category and Cost

\begin{tabular}{lccc}
\hline Category & Establishments & Localization Index & Dispersion Index \\
\hline \$ Shopping & 671 & 0 & 0 \\
$\$ \$$ Shopping & 2511 & 0.0003 & 0 \\
\hline \$ Food & 1384 & 0.0007 & 0 \\
$\$ \$$ Food & 1111 & 0 & 0 \\
\hline \$ Restaurants & 3431 & 0 & 0.0003 \\
$\$ \$$ Restaurants & 2471 & $<.0001$ & 0 \\
\hline
\end{tabular}


Table 5: Location Patterns by Subcategory and Cost

\begin{tabular}{lccc}
\hline Subcategory (category) & Establishments & Localization Index & Dispersion Index \\
\hline Coffee \& Tea (food) & 551 & 0.0025 & 0 \\
$\$$ Coffee \& Tea & 339 & 0.0091 & 0 \\
$\$ \$$ Coffee \& Tea & 193 & 0 & 0 \\
\hline Grocery (food) & 509 & 0 & 0.0014 \\
\$ Grocery & 196 & 0 & 0 \\
$\$ \$$ Grocery & 286 & 0 & 0.0090 \\
\hline Fashion (shopping) & 1007 & 0.0080 & 0 \\
$\$$ Fashion & 190 & 0 & 0 \\
$\$ \$$ Fashion & 510 & 0.0027 & 0 \\
\hline Drugstores (shopping) & 327 & 0 & 0.0212 \\
$\$$ Drugstores & 74 & 0 & 0.0145 \\
$\$ \$$ Drugstores & 229 & 0 & 0 \\
\hline Mexican (restaurants) & 1007 & 0.0080 & 0 \\
$\$$ Mexican & 698 & 0.0029 & 0 \\
$\$ \$$ Mexican & 267 & 0.0013 & 0 \\
\hline American Traditional (restaurants) & 693 & 0 & 0 \\
$\$$ American Traditional & 241 & 0 & \\
$\$ \$$ American Traditional & 422 & 0.0003 & \\
\hline
\end{tabular}


Table 6: Location Patterns by Quality Rating

\begin{tabular}{lccc}
\hline Category & Establishments & Localization Index & Dispersion Index \\
\hline Low Quality Food & 174 & 0 & 0 \\
Medium Quality Food & 1074 & 0 & $<.0001$ \\
High Quality Food & 1413 & 0.0050 & 0 \\
\hline Low Quality Restaurants & 556 & 0 & 0 \\
Medium Quality Restaurants & 3300 & 0 & 0.0086 \\
High Quality Restaurants & 2407 & 0.0198 & 0 \\
\hline Low Quality Shopping & 337 & 0 & 0.0210 \\
Medium Quality Shopping & 1885 & 0 & 0.0201 \\
High Quality Shopping & 2215 & 0.0276 & 0 \\
\hline
\end{tabular}

